\title{
Anapoles and Flying Doughnuts
}

\author{
Nikitas Papasimakis ${ }^{1}$, Vassili Savinov ${ }^{1}$, Apostolos Zdagkas ${ }^{1}$, Nikolay I. Zheludev ${ }^{1,2}$ \\ ${ }^{1}$ Centre for Photonic Metamaterials \& Optoelectronics Research centre, Zepler institute, University of \\ Southampton, Southampton, SO17 1BJ, UK \\ ${ }^{2}$ Centre for Disruptive Photonic Technologies, School of Physical and Mathematical Sciences and The \\ Photonics Institute, Nanyang Technological University, 637371, Singapore
}

\begin{abstract}
We report on recent developments in the light-matter interactions of anapoles and single-cycle toroidal pulses, termed Flying Doughnuts.
\end{abstract}

Toroidal excitations can exist both in matter, as represented by the toroidal multipoles, as well as in freespace in the form of "Flying Doughnuts". Toroidal multipoles provide significant contributions to the electromagnetic response of matter and together with the conventional electric/magnetic multipoles can lead to non-radiating configurations, termed anapoles. On the other hand, Flying Doughnuts are fewcycle electromagnetic pulses with non-trivial spatiotemporal coupling and toroidal configuration of electromagnetic fields that propagate in free-space at the speed of light. They are exact solutions to Maxwell's equations and exhibit strong longitudinal field components along the propagation direction. The spatial and temporal dependence of the Flying Doughnut pulse cannot be separated from one another, which results in a spatially varying frequency spectrum. This spatiotemporal coupling in combination with the doughnut-like arrangement of electromagnetic fields, leads to a complex topological structure in the form of spectrally broadband vortices. Flying Doughnut pulses can interact with matter in unique ways resulting in non-trivial field transformations upon reflection from perfectly conducting and dielectric interfaces, while their interaction with dielectric particles can lead to the excitation of toroidal resonances and non-radiating configurations. Here, we discuss the generation and light-matter interactions of Flying Doughnut pulses with a particular focus on their topological and spatiotemporal structure. We consider the implications of this complex structure for the excitation and detection of toroidal modes and non-radiating configurations in matter. 